\title{
Improved velocity potential formulations of highly accurate Boussinesq-type models
}

Bingham, Harry B.; Engsig-Karup, Allan Peter; Fuhrman, David R.; Madsen, Per A.

Published in:

31. International Conference on Coastal Engineering

Publication date:

2008

Document Version

Early version, also known as pre-print

Link back to DTU Orbit

Citation (APA):

Bingham, H. B., Engsig-Karup, A. P., Fuhrman, D. R., \& Madsen, P. A. (2008). Improved velocity potential formulations of highly accurate Boussinesq-type models. In 31. International Conference on Coastal Engineering (Vol. 1-5, pp. 191-203). American Society of Civil Engineers.

\section{General rights}

Copyright and moral rights for the publications made accessible in the public portal are retained by the authors and/or other copyright owners and it is a condition of accessing publications that users recognise and abide by the legal requirements associated with these rights.

- Users may download and print one copy of any publication from the public portal for the purpose of private study or research.

- You may not further distribute the material or use it for any profit-making activity or commercial gain

- You may freely distribute the URL identifying the publication in the public portal 


\title{
IMPROVED VELOCITY POTENTIAL FORMULATIONS OF HIGHLY ACCURATE BOUSSINESQ-TYPE MODELS.
}

\author{
Harry B. Bingham,, Allan P. Engsig-Karup, David R. Fuhrman, \\ and Per A. Madsen*
}

\begin{abstract}
Several theoretical and numerical aspects concerning the highly accurate Boussinesq-type equations of Madsen et al. (2003, 2006); Jamois et al. (2006) are discussed. A re-derivation of the model recently presented by Bingham et al. (2009) is outlined. This provides a more general framework for the model establishing the correct relationship between a velocity formulation and a velocity potential formulation and correcting previous errors in the potential formulation. A new shoaling enhancement operator is introduced which enables the derivation of new models which differ from the existing ones at $O(\nabla h)$. The performance of the new formulation is validated using computations of linear and nonlinear shoaling problems. The behaviour on a rapidly varying bathymetry is also checked using linear wave reflection from a shelf and Bragg scattering from an undulating bottom. A new stable discretization scheme around structural corners within the fluid domain is also presented.
\end{abstract}

\section{INTRODUCTION}

The most accurate Boussinesq-type formulations for nonlinear water waves yet derived are those originally presented by Madsen et al. $(2002,2003)$ and recently extended to rapidly varying bathymetry by Madsen et al. (2006). These methods achieve superior performance by time-stepping the fully nonlinear free-surface conditions, using a mid-depth expansion level, and retaining the vertical velocity variable as an unknown. Many applications have been presented over the past few years where the method has been used to analyse and elucidate interesting nonlinear wave phenomena. Most of this work has used the original formulation which is in terms of the surface elevation $\eta$, a vertical velocity utility variable $\hat{w}$ and a two-component horizontal velocity utility variable $\hat{\mathbf{u}}$. In the work of Jamois et al. (2006) however, the method was re-cast in terms of a horizontal velocity potential utility

*Department of Mechanical Engineering, Technical University of Denmark, DK-2800 Kgs. Lyngby, Denmark 
variable $\hat{\phi}$ to replace $\hat{\mathbf{u}}$. The motivation for this re-formulation was mainly numerical efficiency as it reduces the size of the linear system by $1 / 3$. The velocity potential formulation used in this work was obtained by ignoring all terms at order $\nabla \hat{z}$ in the flow kinematics (a mild-slope approximation) as was also done in the original formulation. It turns out that although the neglect of these terms does not affect the shoaling properties of the velocity formulation, it completely destroys the performance of the potential formulation. This paper presents a corrected derivation of the method in terms of a utility velocity potential which retains terms proportional to $\nabla \hat{z}$. This allows the potential formulation to achieve a similar shoaling performance to the original velocity formulation. The full details of the new derivation, as well as more complete validation results appear in Bingham et al. (2009).

The second topic of this contribution is concerned with finite difference discretizations of the Boussinesq method around piecewise rectangular structures. A new stable procedure for including such structures within the fluid domain is described. More details on this and other numerical aspects will appear in Bingham and Engsig-Karup (2009).

\section{Formulation}

Consider the irrotational flow of an incompressible inviscid fluid with a free surface. A Cartesian coordinate system is adopted, with the horizontal axes $\mathbf{x}=[x, y]$ located on the still-water plane and the $z$-axis pointing vertically upwards. The fluid domain is bounded by the sea bed at $z=-h(\mathbf{x})$ and the free surface at $z=\eta(\mathbf{x}, t)$. Following Zakharov (1968), the free surface boundary conditions are written in terms of the velocity potential $\tilde{\phi}=$ $\phi(\mathbf{x}, \eta, t)$ and the vertical velocity $\tilde{w}=\left(\phi_{z}\right)_{z=\eta}$ defined directly on the free surface

$$
\begin{gathered}
\eta_{t}+\nabla \eta \cdot \nabla \tilde{\phi}-\tilde{w}(1+\nabla \eta \cdot \nabla \eta)=0 \\
\tilde{\phi}_{t}+g \eta+\frac{1}{2}(\nabla \tilde{\phi})^{2}-\frac{1}{2} \tilde{w}^{2}(1+\nabla \eta \cdot \nabla \eta)=0,
\end{gathered}
$$

where $\nabla=[\partial / \partial x, \partial / \partial y]$ is the horizontal gradient operator and $g$ the gravitational acceleration. Partial differentiation is indicated when the independent variables appear as subscripts. Integrating $\eta$ and $\tilde{\phi}$ forward in time from initial conditions requires a means of computing the associated $\tilde{w}$ such that the flow satisfies the Laplace equation in the fluid domain

$$
\nabla^{2} \phi+\phi_{z z}=0
$$

and the kinematic boundary condition on the sea bottom

$$
w+\nabla h \cdot \nabla \phi=0, \quad z=-h(\mathbf{x}) .
$$

This closure will be solved by means of a Boussinesq-type Taylor series expansion of the velocity potential about an arbitrary level $z=\hat{z}(\mathbf{x})$. 


\section{Boussinesq-type expansion about a rapidly varying level $\hat{z}$}

We outline here the derivation of an infinite series solution for the interior flow problem in terms of a velocity potential. The solution is obtained in a slightly different way than that originally used by Madsen et al. (2002, 2003) and more generally by MFW2006, but the results can be shown to be identical up to first-order in $\delta$ when $\hat{z}(\delta \mathbf{x})$ is a slowly varying function of the horizontal coordinates.

The Taylor series expansion of the solution $\phi(\mathbf{x}, z, t)$ about an arbitrary vertical position $z=\hat{z}(\mathbf{x})$ is given by

$$
\phi(\mathbf{x}, z, t)=\hat{\phi}+\psi \hat{w}+\frac{1}{2} \psi^{2} \hat{\phi}^{(2)}+\frac{1}{6} \psi^{3} \hat{\phi}^{(3)}+\ldots
$$

where

$$
\begin{aligned}
\hat{\phi}=\hat{\phi}^{(0)}=\phi(\mathbf{x}, \hat{z}, t), & \hat{w}=\hat{\phi}^{(1)}=\left.\frac{\partial \phi(\mathbf{x}, z, t)}{\partial z}\right|_{z=\hat{z}} \\
\hat{\phi}^{(n)}=\left.\frac{\partial^{n} \phi(\mathbf{x}, z, t)}{\partial z^{n}}\right|_{z=\hat{z}}, & \text { for } n=2,3, \ldots, \infty .
\end{aligned}
$$

and $\psi=z-\hat{z}$ has been introduced for brevity. Two derivatives of $\phi$ are now taken in each of the three coordinate directions and the result inserted into the Laplace equation. Coefficients are then collected for each power of $\psi$ and set individually to zero to obtain the following recursion relation for $\hat{\phi}^{(n)}$

$$
\begin{array}{r}
\hat{\phi}^{(n)}=\frac{1}{1+\nabla \hat{z}^{2}}\left(-\nabla^{2} \hat{\phi}^{(n-2)}+2 \nabla \hat{z} \cdot \nabla \hat{\phi}^{(n-1)}+\nabla^{2} \hat{z} \hat{\phi}^{(n-1)}\right), \\
n=2,3, \ldots, \infty .
\end{array}
$$

This is a generalisation of the well known constant expansion level recursion relation to an arbitrary level $\hat{z}$. Successive solution of this relation for $n=2,3, \ldots$ provides the exact infinite series solution in terms of $\hat{\phi}$ and $\hat{w}$. The number of terms involved increases rapidly with $n$ however, and a straightforward truncation of the series corresponds to assuming that the spatial variations of $\hat{z}$ and $(\hat{\phi}, \hat{w})$ are similar, which is not necessarily the case. Instead we will assume that $\hat{z}$ is a slowly varying function of the horizontal coordinate to obtain a more convenient solution for practical implementation.

\section{The slowly varying $\hat{z}$ solution}

To simplify the solution, we insert $\hat{z}(\delta \mathbf{x})$ into (7), assuming that $\delta \ll 1$, and collect terms at each order of $\delta$. Dropping terms above $O(\delta)$ in the recursion relation and solving successively for $n=2,3, \ldots, \infty$ we express each $\hat{\phi}^{(n)}$ in 
terms of $\hat{\phi}$ and $\hat{w}$. With these solutions inserted into (5) the following $O(\delta)$ solution is obtained

$$
\begin{array}{cc}
\phi(\mathbf{x}, z, t)= & \operatorname{Cos}(\psi \nabla) \hat{\phi}+\operatorname{Sin}(\psi \nabla) \nabla^{-1} \hat{w} \\
& +\nabla \hat{z} \cdot\left[\left(\psi \operatorname{Cos}(\psi \nabla)-\operatorname{Sin}(\psi \nabla) \nabla^{-1}\right) \nabla \hat{\phi}+\psi \operatorname{Sin}(\psi \nabla) \nabla^{-1} \nabla \hat{w}\right] .
\end{array}
$$

This compact form is obtained by associating each infinite series operator with the Taylor series expansion of an irrational function. For example, the first two infinite series operators appearing here are

$$
\begin{aligned}
\operatorname{Cos}(\psi \nabla) & \equiv 1-\frac{1}{2} \psi^{2} \nabla^{2}+\frac{1}{24} \psi^{4} \nabla^{4}+\ldots \\
\operatorname{Sin}(\psi \nabla) \nabla^{-1} & \equiv \psi-\frac{1}{6} \psi^{3} \nabla^{2}+\frac{1}{120} \psi^{5} \nabla^{4}+\ldots
\end{aligned}
$$

The convention adopted here is to express all infinite series operators in terms of even (hence scalar) derivatives which should be understood to act independently on each component when applied to a vector. Thus e.g. $\nabla^{2} \nabla \hat{w} \equiv\left[\nabla^{2} \hat{w}_{x}, \nabla^{2} \hat{w}_{y}\right]$

The fluid velocities are now given by derivatives of (8). This potential formulation can be converted to an equivalent velocity formulation using the relation $\nabla \hat{\phi}=\hat{\mathbf{u}}+\nabla \hat{z} \hat{w}$. This manipulation recovers the corresponding Method I expressions obtained by Madsen et al. $(2006,2003)$ up to first order in $\delta$.

\section{Dispersion and shoaling enhancement}

The accuracy of the truncated approximation can be significantly improved by applying an enhancement technique to the differential operators. This is done by defining an enhancement operator $L(\hat{z} \nabla)$, along with new (nonphysical) expansion variables $\hat{\phi}^{*}, \hat{w}^{*}$ such that

$$
\hat{\phi}=L_{p}(\hat{z} \nabla) \hat{\phi}^{*}, \quad \hat{w}=L_{w}(\hat{z} \nabla) \hat{w}^{*} .
$$

In previous work only one dispersion enhancement operator was used:

$$
L_{0}(\hat{z} \nabla)=\sum_{n=0}^{2 N} \lambda_{2 n} \hat{z}^{2 n} \nabla^{2 n}
$$

with coefficients $\lambda_{i}$ obtained by setting to zero all terms with powers of $2 N+2$ to $4 N+1$ in the product of $L_{0}$ and the Taylor series expansions up to order $4 N+1$ of the operators $\operatorname{Sin}(\hat{z} \nabla)$ and $\operatorname{Cos}(\hat{z} \nabla)$. The goal here is to double the accuracy of the truncated expansions, giving the resultant dispersion operator a Padé expansion form. The first two such operators 
are

$$
\begin{array}{lll}
L_{0}(\hat{z} \nabla)= & 1+\frac{(\hat{z} \nabla)^{2}}{10}+\frac{(\hat{z} \nabla)^{4}}{120}, & N=1 \\
L_{0}(\hat{z} \nabla)= & 1+\frac{(\hat{z} \nabla)^{2}}{18}+\frac{(\hat{z} \nabla)^{4}}{504}+\frac{(\hat{z} \nabla)^{6}}{15120}+\frac{(\hat{z} \nabla)^{8}}{362880}, & N=2 .
\end{array}
$$

Here we extend the idea by defining two additional shoaling enhancement operators such that

$$
\begin{array}{cl}
L_{p}=L_{0}+\nabla \hat{z} \cdot L_{1} \nabla, & L_{w}=L_{0}+\nabla \hat{z} \cdot L_{2} \nabla \\
L_{1}(\hat{z} \nabla)=\sum_{n=1}^{N+1} a_{2 n-1} \hat{z}^{2 n-1} \nabla^{2 n-2}, & L_{2}(\hat{z} \nabla)=\sum_{n=1}^{N+1} b_{2 n-1} \hat{z}^{2 n-1} \nabla^{2 n-2}
\end{array}
$$

The coefficients $a_{i}, b_{i}$ appearing in the new shoaling enhancement operators $L_{1}$ and $L_{2}$ are then chosen to optimise the linearised shoaling behaviour of the resultant method.

The enhanced definition of the expansion variables (10) is now inserted into the flow solution (8). When the series operators in this equation act on the $L$-operator they modify the coefficients of the original series as well as generating new terms. The new terms are collected into powers of $\delta$ and terms at order $\delta^{2}$ and higher are dropped. The final result has the form

$$
\phi(\mathbf{x}, z, t)=\left(J_{01}+\delta \nabla \hat{z} \cdot J_{11 p} \nabla\right) \hat{\phi}^{*}+\left(J_{02}+\delta \nabla \hat{z} \cdot J_{12 p} \nabla\right) \hat{w}^{*} .
$$

The fluid velocities are obtained by differentiating (15) and this leads to a set of consistency relations between all the operators (see Bingham et al. (2009)) which provide a convenient check that mass conservation is achieved to the truncation order of the scheme. Analogous mass conservation constraints for a velocity formulation are also given there.

To obtain a truncated method for practical implementation, we apply the above described enhancement strategy to (8) and it's derivatives to derive all of the $J$-operators, retaining derivatives at least up to $2 N+2$. All operators acting on $\hat{\phi}$ are then truncated at derivative order $2 N+2$ while those operating on $\hat{w}$ are truncated at $2 N+1$. The resultant $N=1$ and $N=2$ operators are rather lengthy and not given here but can be found in Bingham et al. (2009). In that reference, the $N=2$ enhanced potential formulation is also shown to recover the original velocity formulation (without shoaling optimisation) when converted to a velocity formulation.

Analysis of linear shoaling The performance of this new model with respect to linear shoaling is shown in Figure 1 where it is compared to the exact solution from linear theory. Three model results appear in this figure. The curve marked " $O(0)$ " shows the model without $\nabla \hat{z}$ terms which was discussed by Jamois et al. (2006). This curve shows the behaviour of the $O(0)$ in $\delta$ model without any attempt to optimise for linear shoaling, 

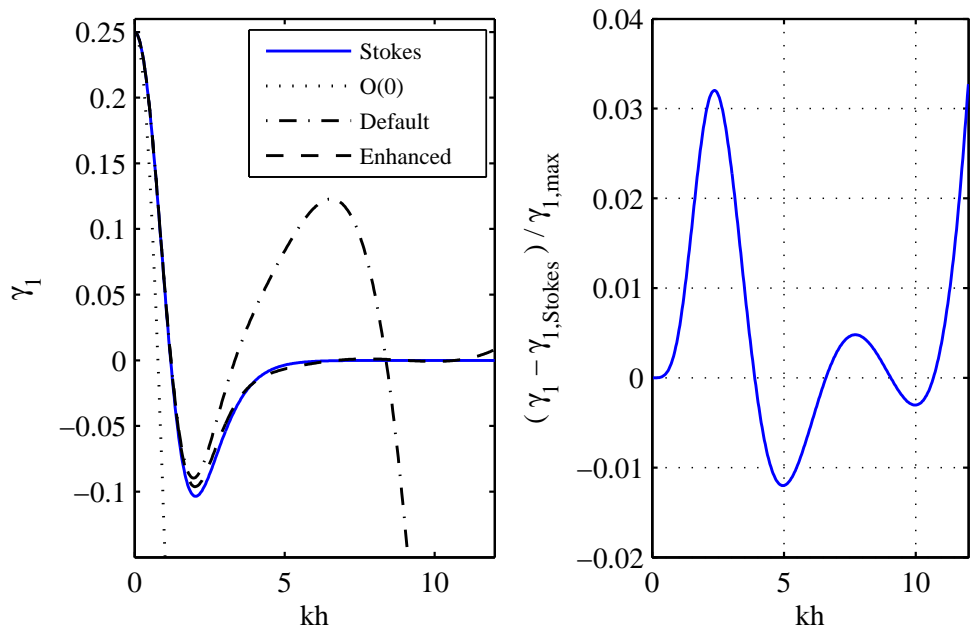

Figure 1: Left: The linear shoaling gradient for the $N=1$ model. $O(0)$ is the model used by Jamois et al. (2006). The other two curves show the new $O(\delta)$ model with and without using the shoaling enhancement operators $L_{1}$ and $L_{2}$. Right: Relative error for the enhanced model.

but we have so far found no way to significantly improve the result without including terms at $O(\delta)$. Thus, the claimed ability to shoal waves out to $\kappa=10$ with the potential formulation of Jamois et al. (2006) is incorrect. The optimised coefficients given there were in fact obtained from the $O(0)$ in $\delta$ velocity formulation by incorrectly assuming that the two formulations behaved in the same way with respect to linear shoaling. The curve marked "Default" is the new $O(\delta)$ model without the shoaling enhancement operators $L_{1}$ and $L_{2}$, i.e. setting all the $a_{i}, b_{i}$ coefficients to zero. The curve marked "Enhanced" is obtained by using the values listed by Bingham et al. (2009) which have been determined via minimisation of the shoaling errors over the range $0 \leq \kappa_{0} \leq 10$ with $\hat{z}=-h / 2$.

\section{Numerical solution using finite-differences}

A general purpose, finite difference based, numerical solution of the above described Boussinesq-type model has been implemented in nearly the same manner as that described in detail by Fuhrman and Bingham (2004); Fuhrman et al. (2004); Jamois et al. (2006). The new implementation improves on earlier solutions in several ways which will be described in detail by Bingham and Engsig-Karup (2009). Here we briefly summarise the numerical discretization procedure and describe the new method for inserting piecewise rectangular structures into the fluid domain. 

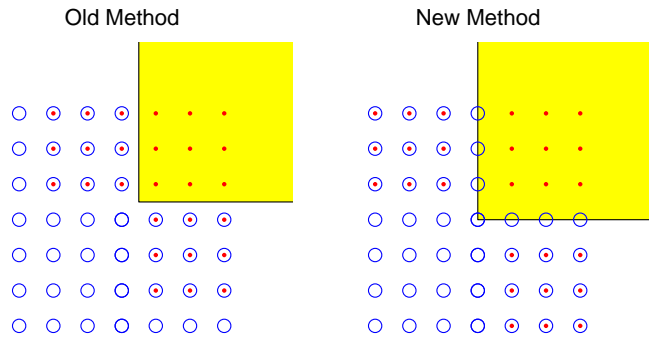

Figure 2: The old and new discretization procedures around structural corners within the fluid domain. Open circles show grid points within the fluid domain and the solid circles show how coefficients are reflected about the boundary when using a 7-point stencil in each direction.

A structured grid of $N_{x}$ by $N_{y}$ points is distributed over the rectangular still water plane. One arbitrarily spaced set of points is taken along each coordinate direction, and 1D finite difference operators of order $r-1$ are developed at each point for the first four or six derivatives using Taylor series expansion. The point where the derivative is desired is always included, so $r$ is an odd integer. To maintain a consistent order of accuracy, $r$ points are used for first- and second-derivatives, but $2[(r-1) / 2+1]+1$ points for thirdand fourth-derivatives, etc. Homogeneous Neumann boundary conditions at the side walls of the domain are imposed by reflecting the finite-difference coefficients for a function which is symmetric about the boundary, thus all schemes are effectively centred. 2D cross-derivative operators are then obtained via multiplication of the $1 \mathrm{D}$ discrete operators (successive application of the schemes.)

Equations (1) and (2) are evolved forward from initial conditions at each grid point using the classical explicit fourth-order Runge-Kutta scheme. All terms on the right hand side of these equations can be directly computed using the above described finite-difference operators, except $\tilde{w}$. The Boussinesq model is applied to find this quantity by expressing $\tilde{\phi}$ using (15) at $z=\eta$, and the bottom boundary condition (4). This leads to a sparse linear system of equations of rank $2 n$ where $n=N_{x} N_{y}$. This system is solved using the GMRES iterative scheme, preconditioned on the left by an incomplete LU-factorisation of the linearised, lowest-order accurate version of the system matrix as discussed in detail by Fuhrman and Bingham (2004). This gives $\hat{\phi}^{*}$ and $\hat{w}^{*}$ at which point $\tilde{w}$ is computed from the z-derivative of (15) applied at $z=\eta$ to close the problem.

New discretization procedure around corners In Fuhrman et al. (2005); Jamois et al. (2006) piecewise rectangular structures extending uniformly through the depth of the fluid were introduced into the fluid domain 
by simply reflecting the finite difference coefficients about these boundaries. In that work, the boundaries were taken to lie midway between grid points as shown in the left-hand plot in Figure 2. This led to an ambiguity about how to reflect coefficients for grid points near external corners (i.e. first in $x$ and then in $y$ or the reverse), and an average of the two possibilities was taken. The resultant numerical scheme turned out to be generally unstable (more and more so as the problem became more and more nonlinear) and often required large amounts of filtering near external corners. The new discretization scheme shown to the right in this figure, has no negative effect on the stability of the overall method. In this scheme, the boundaries and corners are taken to lie on the grid points. Discrete 1D derivative operators are then developed in each coordinate direction where there is no ambiguity about reflection of the coefficients. Finally, mixed-derivative operators are obtained by multiplication of the uni-directional operators. As these 1D derivative operators do not in general commute, we take the average of the two possible products.

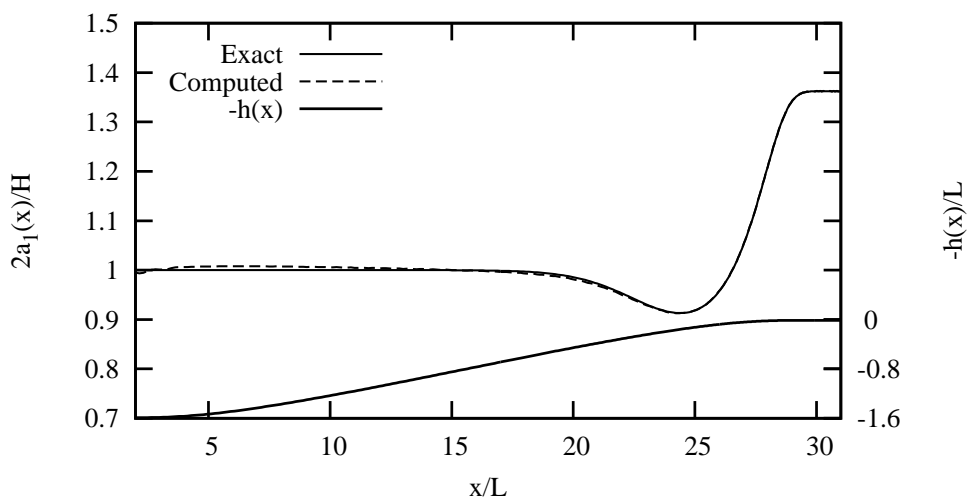

Figure 3: Linear shoaling wave elevation amplification factor on a smooth beach geometry with average slope of $5 \%$ covering the range $10 \geq k h \geq .25$.

\section{Model calculations}

In this section we present numerical calculations for several test cases of wave transformation on a variable depth fluid. More extensive applications can be found in Bingham et al. (2009); Bingham and Engsig-Karup (2009). Sixth-order accurate finite difference approximations and a Courant number $C_{r}=L / T \Delta t / \Delta x_{\text {ave }}=1 / 2$ been used for all calculations, where $\Delta x_{\text {ave }}$ is the average grid spacing and $L / T$ the deep water wave speed. Wave 


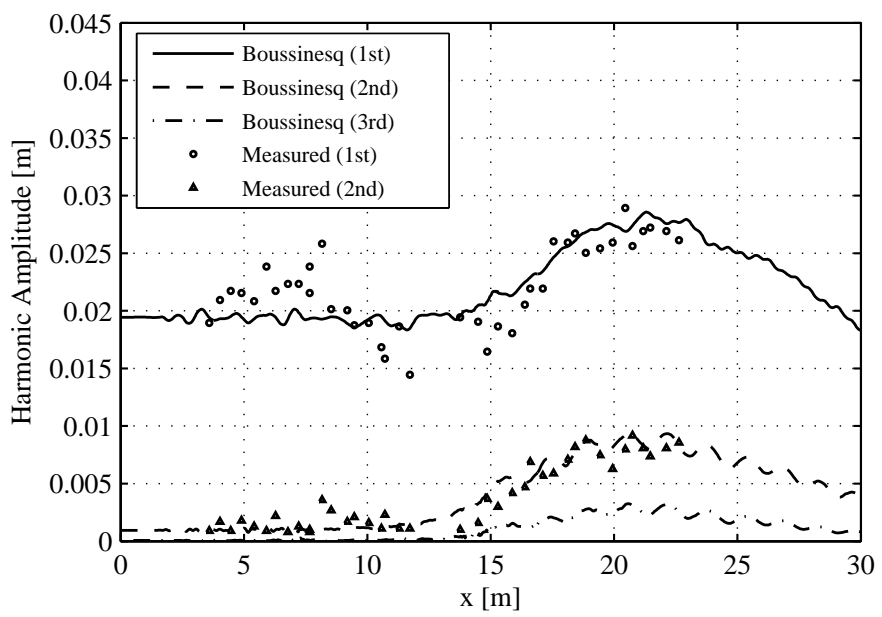

Figure 4: Nonlinear waves on a semi-circular shoal, $T=1$ s case. $N_{x}=$ 798, $N_{y}=53, C_{r}=1 / 2$. Harmonic amplitudes along the tank centre line.

generation and absorption is done using relaxation-zones of approximately two wavelengths.

Linear shoaling on a mildly sloping beach In order to confirm the analysis of the linear shoaling properties of the model we consider the evolution of very small amplitude waves on a plane beach of mild slope. The smooth beach profile shown in Figure 3 provides local relative depths running from $10 \geq k h \geq .25$ and a maximum slope of approximately $8 \%$. The deep water wavelength is $L$ and the domain is of length $32 \mathrm{~L}$. At the shallow end the wavelength has reduced to $L_{1}=.28 L . N_{x}=443$ grid points have been used, distributed along the tank based on the local water depth to give approximately eight to ten grid points per wavelength everywhere in the domain. These calculations were run until a steady-state was reached and the local wave amplitude amplification factor (normalised by the deep water wave height $H$ ) is compared to the exact result from linear theory in Figure 3. The errors can be seen to be small and generally consistent with the analysis shown in Figure 1.

Nonlinear waves on a semi-circular shoal To test the performance of the model for nonlinear refraction-diffraction problems we consider the case investigated experimentally by Whalin (1971). Nearly linear plane waves are incident from the left and encounter a semi-circular shoaling region and subsequent shelf. The semi-circular shoal region causes the waves to refract, steepen and focus, finally releasing higher harmonics on the shelf to create an 
irregular, short-crested 3D pattern. We show here results from the case with $T=1 \mathrm{~s}$, and $H=0.0390 \mathrm{~m}$. A harmonic analysis of the steady-state time series along the centre line of the tank is shown in Figure 4. A uniform grid spacing has been applied, with the number of grid points chosen to ensure that the free third-harmonic wave is resolved with four to six grid points per wavelength on the shallow shelf. The comparison with the measured data is generally good.

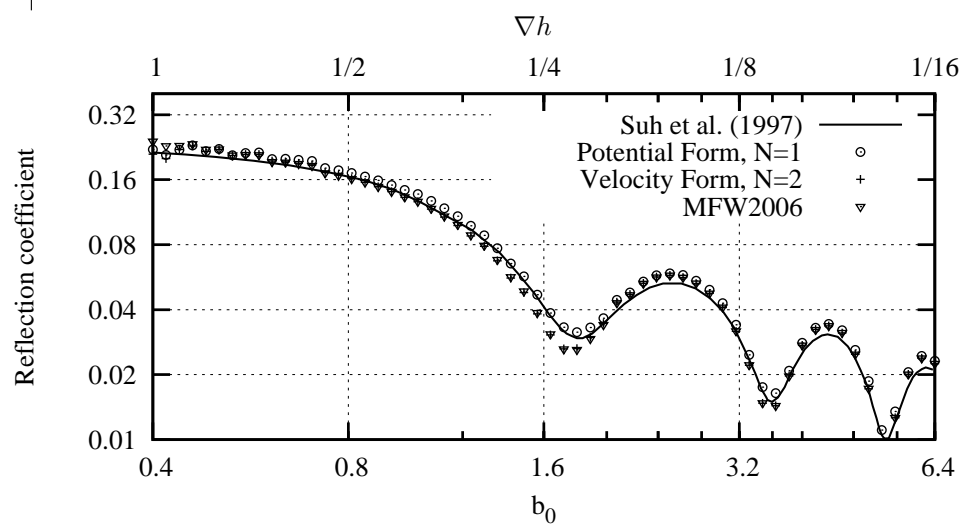

Figure 5: Reflection coefficient of linear shallow water waves from a plane step plotted vs. step length.

Reflection from a plane slope We now test the performance of the model on a rapidly varying bathymetry by considering the reflection of linear monochromatic waves from a plane slope and subsequent shelf. The first case, originally suggested by Booij (1983), concerns two constant depth regions at $h=0.6 \mathrm{~m}$ and $h=0.2 \mathrm{~m}$ connected by a plane slope of width $b_{0}$. The width $b_{0}$ ranges from $6.4 \mathrm{~m}$ to $0.4 \mathrm{~m}$ corresponding to slopes of $1 / 16$ to 1. A linear wave of period $T=2 \mathrm{~s}$ is incident from the deep water region for each case which gives relative water depths of $k h=.9$ and $k h=.4$ in the deep and shallow regions, thus a relatively shallow test case. We take $\hat{z}=$ $-.3 h$ to the left and $\hat{z}=-.45 h$ to the right of the slope, joining these values with a straight line, and smoothing the result. The grid spacing is taken to be uniform with $\Delta x=0.04 \mathrm{~m}$. Figure 5 compares the resultant reflection coefficients with the solutions of Suh et al. (1997) who used the extended mild-slope equation. We conclude from this that the new methods can treat shallow water reflection problems up to bottom slopes of approximately one.

The second case considers the same problem but in intermediate to deep water. The geometry consists of a a deep region with $h_{2}=20 \mathrm{~m}$ and a shallow region with $h_{1}=5 \mathrm{~m}$, connected by a steep plane with slope 0.5 . For the 


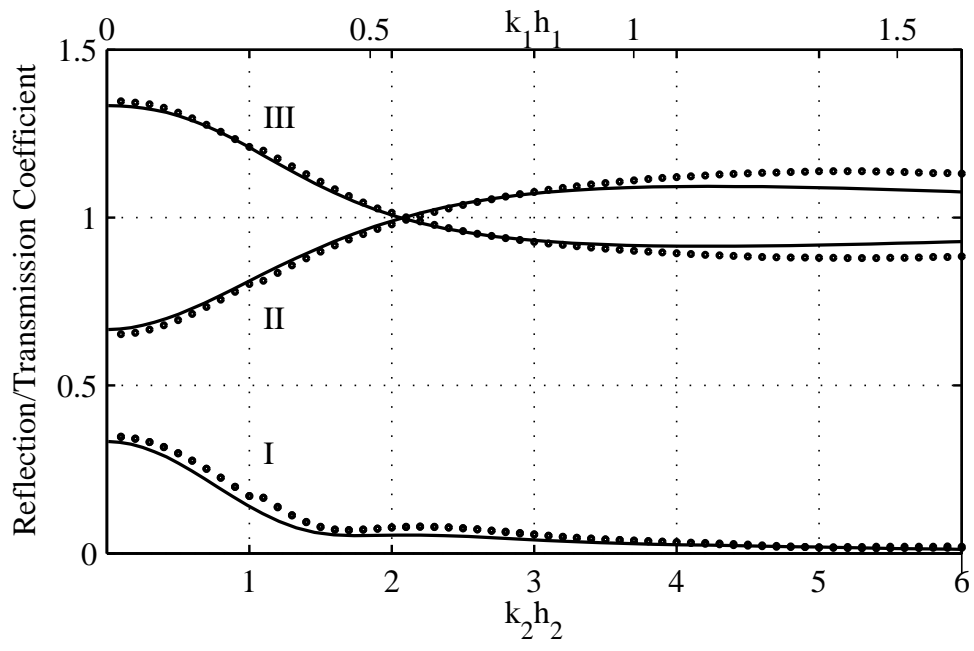

Figure 6: Reflection/transmission of linear intermediate to deep water waves on a steep step of slope 0.5. I - Reflection coefficient, II - Transmission from shallow to deep water; and III - Transmission from deep to shallow water. Lines are the calculations using Bender and Dean (2003), points are the current calculations.

intermediate to deep water cases with $k_{2} h_{2}>1$ we set $\hat{z}=-h / 2$ at the deep end and $\hat{z}=-.45 h$ at the shallow end, connect them with a straight line, and smooth the result. The incoming waves may travel in either direction, and the wave period is varied to cover the range $0.1 \leq k_{2} h_{2} \leq 6$ corresponding to $.05<k_{1} h_{1}<1.62$. Figure 6 compares the resultant reflection and transmission coefficients with calculations using the method of Bender and Dean (2003) who used a step-wise modal decomposition approach. The agreement is reasonably good.

Bragg scattering from an undulating bottom As a final example, we consider the reflection of plane waves from an undulating bottom. The case considered here is for Class I Bragg resonance in $2 \mathrm{D}$ for which the resonance condition $k_{1}=-k_{2}=\frac{K}{2}$, and $\omega_{1}=\omega_{2}$. where $k_{1}, k_{2}$, and $K$ are the incident, reflected, and bottom wave numbers respectively, and $\omega_{1}, \omega_{2}$ are the corresponding frequencies. Calculations are compared with the experimental measurements of Davies and Heathershaw (1984) for the case with ten bottom ripples at $K d=0.31$ and $d / h_{0}=0.16$, with the bottom defined by $h(x)=h_{0}+d \sin (K x)$ over the ripple patch and by $h=h_{0}$ elsewhere. A constant grid spacing corresponding to twenty points per bottom wave length have been used with $\hat{z}=-.5 h_{0}$. The comparison 


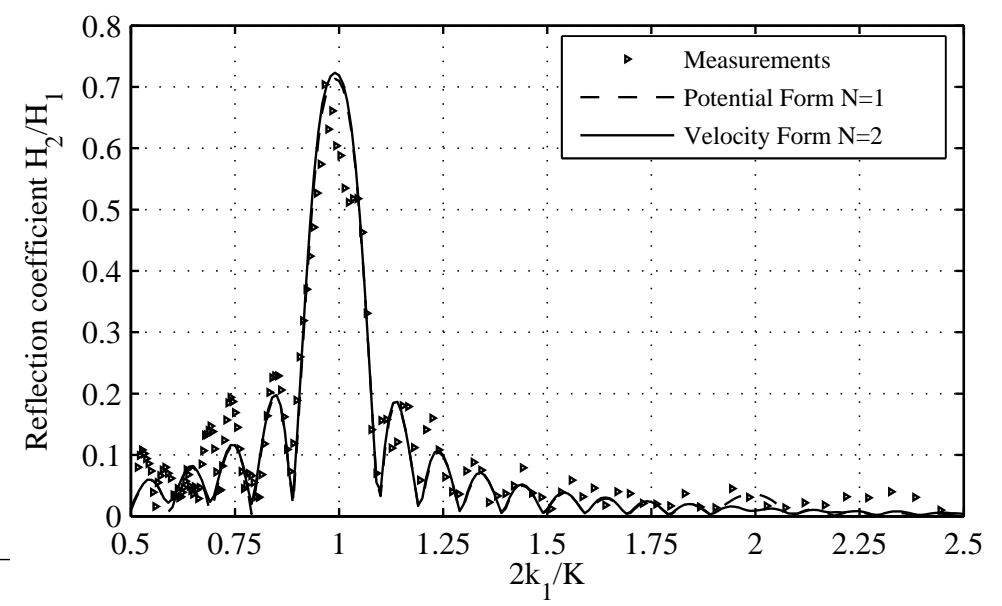

Figure 7: Reflection of linear waves from a sinusoidal bottom ripple patch with $K d=.31, d / h=.16$ and 10 ripples. $\Delta x=2 \pi / K / 20, C_{r}=1 / 2$.

is shown in Figure 7, and the agreement is good.

\section{Acknowledgements}

We are grateful for funding from the Danish Research Council for Technology and Production grant no. 274-06-0030 and for supercomputing resources made available by the Danish Centre for Scientific Computing.

\section{References}

Bender, C. J., Dean, R. G., 2003. Wave transformation by two-dimensional bathymetric anomalies with sloped transitions. Coastal Engineering 50, 61-84.

Bingham, H. B., Engsig-Karup, A. P., 2009. High accuracy Boussinesq methods for wave-structure interaction (In preparation).

Bingham, H. B., Madsen, P. A., R., F. D., 2009. Velocity potential formulations of Boussinesq-type models. Coastal Engineering To Appear.

Booij, N., 1983. A note on the accuracy of the mild-slope equations. Coastal Engineering 7, 191-203. 
Davies, A. G., Heathershaw, A. D., 1984. The reflection of wave energy by undulations on the seabed. J. Fluid Mech. 144, 419-443.

Fuhrman, D. R., Bingham, H. B., 2004. Numerical solutions of fully nonlinear and highly dispersive Boussinesq equations. Int. J. Num. Methods in Fluids 44 (3), 231-255.

Fuhrman, D. R., Bingham, H. B., Madsen, P. A., 2005. Nonlinear wavestructure interaction with a high-order Boussinesq model. Coastal Engineering 52, 655-672.

Fuhrman, D. R., Bingham, H. B., Madsen, P. A., Thomsen, P. G., 2004. Linear and non-linear stability analysis for finite difference discretizations of high-order Boussinesq equations. Int. J. Num. Methods in Fluids 45 (7), 751-774.

Jamois, E., Fuhrman, D. R., Bingham, H. B., Molin, B., 2006. Wavestructure interactions and nonlinear wave processes on the weather side of reflective structures. Coastal Engineering 53, 929-945.

Larsen, J., Dancy, H., 1983. Open boundaries in short wave simulations - a new approach. Coastal Engineering 7, 285-297.

Madsen, P. A., Bingham, H. B., Liu, H., 2002. A new Boussinesq method for fully nonlinear waves from shallow to deep water. J. Fluid Mech. 462, $1-30$.

Madsen, P. A., Bingham, H. B., Schäffer, H. A., 2003. Boussinesq-type formulations for fully nonlinear and extremely dispersive water waves: Derivation and analysis. Proc. R. Soc. Lond. A 459, 1075-1104.

Madsen, P. A., Fuhrman, D. R., Wang, B., 2006. A Boussinesq-type method for fully nonlinear waves interacting with a rapidly varying bathymetry. Coastal Engineering 53, 487-504.

Suh, K. D., Lee, C., Park, W. S., 1997. Time-dependent equations for wave propgation on rapidly varying topography. Coastal Engineering 32, 91117.

Whalin, R. W., 1971. The limit of applicability of linear wave refraction theory in a convergence zone. Tech. Rep. Res. Rep. H-71, U.S. Army Corps of Engineers, Waterways Expt. Station, Vicksburg, MS, U.S.A. 